\title{
RESEARCH
}

Open Access

\section{Enantioselective metabolism of primaquine by human CYP2D6}

Pius S Fasinu', Babu L Tekwani ${ }^{1,2}$, NP Dhammika Nanayakkara', Bharathi Avula ${ }^{1}$, HMT Bandara Herath', Yan-Hong Wang ${ }^{1}$, Vijender R Adelli ${ }^{1}$, Mahmoud A Elsohly ${ }^{1,3,4}$, Shabana I Khan ${ }^{1,2}$, Ikhlas A Khan ${ }^{1,2}$, Brandon S Pybus ${ }^{5}$, Sean R Marcsisin ${ }^{5}$, Gregory A Reichard ${ }^{5}$, James D McChesney ${ }^{6}$ and Larry A Walker ${ }^{1,2^{*}}$

\begin{abstract}
Background: Primaquine, currently the only approved drug for the treatment and radical cure of Plasmodium vivax malaria, is still used as a racemic mixture. Clinical use of primaquine has been limited due to haemolytic toxicity in individuals with genetic deficiency in glucose-6-phosphate dehydrogenase. Earlier studies have linked its therapeutic effects to CYP2D6-generated metabolites. The aim of the current study was to investigate the differential generation of the CYP2D6 metabolites by racemic primaquine and its individual enantiomers.

Methods: Stable isotope ${ }^{13} \mathrm{C}$-labelled primaquine and its two enantiomers were incubated with recombinant cytochrome-P450 supersomes containing CYP2D6 under optimized conditions. Metabolite identification and time-point quantitative analysis were performed using LC-MS/MS. UHPLC retention time, twin peaks with a mass difference of 6, MS-MS fragmentation pattern, and relative peak area with respect to parent compound were used for phenotyping and quantitative analysis of metabolites.
\end{abstract}

Results: The rate of metabolism of (+)-(S)-primaquine was significantly higher (50\% depletion of $20 \mu \mathrm{M}$ in $120 \mathrm{~min}$ ) compared to $(-)-(R)$-primaquine (30\% depletion) when incubated with CYP2D6. The estimated $V_{\max }$ $\left(\mu \mathrm{mol} / \mathrm{min} / \mathrm{mg}\right.$ ) were $0.75,0.98$ and 0.42 , with $K_{m}(\mu \mathrm{M})$ of $24.2,33.1$ and 21.6 for $( \pm)$-primaquine, $(+)$-primaquine and (-)-primaquine, respectively. Three stable mono-hydroxylated metabolites, namely, 2-, 3- and 4-hydroxyprimaquine (2-OH-PQ, 3-OH-PQ, and 4-OH-PQ), were identified and quantified. 2-OH-PQ was preferentially formed from $(+)$-primaquine in a ratio of 4:1 compared to (-)-primaquine. The racemic $( \pm)$-primaquine showed a pattern similar to the (-)-primaquine; 2-OH-PQ accounted for about 15-17\% of total CYP2D6-mediated conversion of (+)-primaquine. In contrast, 4-OH-PQ was preferentially formed with (-)-primaquine (5:1), accounting for $22 \%$ of the total (-)-primaquine conversion. 3-OH-PQ was generated from both enantiomers and racemate. 5-hydroxyprimaquine was unstable. Its orthoquinone degradation product (twice as abundant in (+)-primaquine compared to (-)-primaquine) was identified and accounted for 18-20\% of the CYP2D6-mediated conversion of (+)-primaquine. Other minor metabolites included dihydroxyprimaquine species, two quinone-imine products of dihydroxylated primaquine, and a primaquine terminal alcohol with variable generation from the individual enantiomers.

Conclusion: The metabolism of primaquine by human CYP2D6 and the generation of its metabolites display enantio-selectivity regarding formation of hydroxylated product profiles. This may partly explain differential pharmacologic and toxicologic properties of primaquine enantiomers.

Keywords: Primaquine enantiomers, CYP2D6, Metabolism, Ring-hydroxylation, Quinone-imines, Orthoquinone

\footnotetext{
* Correspondence: Iwalker@olemiss.edu

${ }^{1}$ The National Center for Natural Products Research, University of Mississippi, University, MS 38677, USA

${ }^{2}$ BioMolecular Sciences, University of Mississippi, University, MS 38677, USA

Full list of author information is available at the end of the article
} 


\section{Background}

Primaquine, a prototype 8-aminoquinoline, the only licensed option to treat the relapsing liver stages (hypnozoites) of Plasmodium vivax, is also used for the prophylaxis of all forms of human malaria [1-4]. Due to its activity against mature, infective Plasmodium falciparum gametocytes, this drug can be employed clinically as a gametocytocide for blocking transmission in P. falciparum malaria. In areas of emerging drug resistance, primaquine has shown effectiveness against and in the prevention of the spread of artemisinin-resistant $P$. falciparum strains [5]. Since its introduction into the market in the 1950s, primaquine has been documented to trigger haemolysis in individuals with a genetic deficiency in glucose-6-phosphate dehydrogenase (G6PD) [3,4]. Thus, despite the unique therapeutic indications of primaquine, the widespread prevalence of G6PD deficiency across populations in malaria-endemic areas has limited its clinical use [6,7].

In 1962, Tarlov and co-workers suggested that a metabolite of primaquine, rather than primaquine itself, might be responsible for the haematotoxicity of primaquine [8]. This was based on the observed delay in anticatalase activity in an individual dosed with the drug; it was postulated that redox-cycling of the metabolite 6-demethyl-5-hydroxyprimaquine and its corresponding orthoquinone caused oxidative stress [8]. Even though positive identification in metabolic mixtures could be made only during the last decade [9] due to the high reactivity of the metabolites and limitations of available analytical methods, a number of in vitro studies have shown that 5-hydroxyprimaquine and its oxidative products caused haematotoxicity [10-13]. Carboxyprimaquine, which has been identified as the major circulating metabolite in several species, including man, after oral administration of primaquine [14] has been found to be non-toxic [11] and inactive [15]. An earlier in vitro study suggested that multiple CYP isoforms (CYP2E1, CYP2B6, CYP1A2, CYP2D6, and CYP3A4) variably contributed to the haemotoxic response of primaquine [16]. Monoamine oxidase (MAO) has been shown as responsible for the formation of carboxyprimaquine [17], whereas CYP enzymes, especially CYP2D6, were found to produce several ringhydroxylated primaquine metabolites [18].

Recent work using CYP2D knockout and humanized CYP2D6 mice, highlighted the dependence of the efficacy of primaquine and other 8-aminoquinolines (8AQ) on CYP2D metabolism in a rodent causal prophylaxis model $[19,20]$. The human relevance of this finding was substantiated by the observed treatment failures of primaquine in humans with $P$. vivax malaria who are deficient in CYP2D6 enzyme activity which suggested that CYP2D6 is required for the generation of the active metabolites [21].

Primaquine is a chiral drug and is currently used as a racemic mixture, approximating a 50:50 ratio of $(+)-(S)$ - and $(-)-(R)$-enantiomers. Previously, differential activity, toxicity and pharmacokinetic profiles for the individual enantiomers of primaquine have been shown [22-24]. (+)-(S)-primaquine showed better causal prophylactic and blood schizonticidal activities in Plasmodium berghei mouse malaria models and higher propensity to cause haematotoxicity in a non-obese diabetic/severe combined immunodeficiency (NOD/SCID) mouse model engrafted with G6PD-deficient human erythrocytes and beagle dogs [23]. However, in rhesus monkeys, the results [24] were in agreement with the report by Schmidt et al. [22] that both enantiomers had equivalent radical curative activity against Plasmodium cynomolgi. The stereo-selectivity in metabolite generation and other pharmacokinetic behavior of chiral antimalarial drugs has been known to lead to major differences in pharmacodynamic properties of individual enantiomers [25]. Similarly, the enantioselective pharmacologic and toxicologic properties of primaquine may be attributed to differential pharmacokinetic profiles of the two enantiomers. Studies with mice and humans have shown that the major serum metabolite, carboxyprimaquine, which amounted to more than $60 \%$ of total metabolites was predominantly emanating from $(-)-(R)$-primaquine [26].

Considering the essential contribution of CYP2D6-linked metabolism to the efficacy and toxicity of primaquine, and the generation of multiple quinoline ring-hydroxylated metabolites on incubation of racemic primaquine with CYP2D6, it was hypothesized that observed variation in therapeutic response of the two primaquine enantiomers may be attributed to enantio-selective CYP2D6mediated ring hydroxylation of primaquine. Challengingly, the low quantities and the highly reactive nature of the ring-hydroxylated primaquine metabolites pose major problems regarding phenotyping and quantification of these metabolites. Recently this challenge has been addressed by the application of 50:50 mixture of ${ }^{13} \mathrm{C}$-stable isotope labelled $\left(\mathrm{C}_{6}\right)$ and normal ${ }^{12} \mathrm{C}$ - primaquine followed by analysis with liquid chromatography-mass spectrometry (LC-MS/MS) [9].

For the identification of metabolites, 2-, 3-, 4-, and 5-hydroxyprimaquine (2-OH-PQ, 3-OH-PQ, 4-OH-PQ and $5-\mathrm{OH}-\mathrm{PQ}$ ) and $8-\mathrm{N}$-hydroxyprimaquine were prepared as reference standards. Attempts at preparation of 7-hydroxyprimaquine have been unsuccessful to date. 5-OH-PQ spontaneously underwent oxidation yielding the orthoquinone product.

Comparison of ultra-high performance liquid chromatography (UHPLC) retention times (RT) of twin mass peaks with difference of 6 (originating from ${ }^{13} \mathrm{C}_{6}$ - primaquine $/{ }^{12} \mathrm{C}$ - primaquine) with those of reference standards, MS-MS fragmentation patterns, and relative peak area with respect to parent compound were used 
for phenotyping and semi-quantitative analysis of the metabolites.

\section{Methods}

\section{Synthetic chemicals}

${ }^{13} \mathrm{C}(6)$-labelled primaquine (racemic, and the $(+)-(S)$ - and $(-)-(R)$ - enantiomers) [27] and primaquine alcohol [28] were synthesized as previously reported. The identity of the compounds synthesized was confirmed by spectral infra-red (IR), nuclear magnetic resonance (NMR) and high-resolution MS and physical data in comparison with published values. 4-OH-PQ and 5-OH-PQ were prepared using the previously reported procedures $[29,30]$. The latter underwent rapid spontaneous oxidation. Time-lapse LC-MS analysis indicated formation of quinone-imine, which underwent demethylation and isomerization yielding a stable 5,6-orthoquinone analog. The methods for synthesis of other analogs, which were used as standards for phenotyping and quantification of the primaquine metabolites, are described below.

\section{Synthesis of 2-hydroxyprimaquine diphosphate 2-benzyloxy-6-methoxy-8-(1-methyl-4-phthalimidobutylamino) quinoline}

A mixture of 2-benzyloxy-6-methoxy-8-nitroquinoline [31] (2.75 g) hydrazine hydrate $(3 \mathrm{ml})$ and Raney $\mathrm{Ni}$ $(1.5 \mathrm{~g})$ in ethanol $(75 \mathrm{ml})$ was refluxed for four hours. The catalyst was removed by filtration through celite and the filtrate was evaporated. The residue was partitioned between $\mathrm{CH}_{2} \mathrm{Cl}_{2}$ and water and the organic layer was dried with sodium sulphate and evaporated to give 8-amino-2-benzyloxy-6-methoxyquinoline $(2.3 \mathrm{~g})$. To a stirred mixture of this product $(2.3 \mathrm{~g}, 7.8 \mathrm{~mm})$ and 2-oxo-5-phthalimido pentane $(2.0 \mathrm{~g}, 8.6 \mathrm{~mm})$ in glacial acetic acid $(30 \mathrm{ml})$, sodium borohydride was added portionwise while maintaining the temperature below $30^{\circ} \mathrm{C}$ until reaction was complete evidenced by thin layer chromatography (TLC). The reaction mixture was poured onto ice and basified with aqueous sodium hydroxide (50\%). The product was separated by filtration and purified by chromatography on silica gel with hexanes:ethyl acetate 85:15 to give 2-benzyloxy-6-methoxy-8-(1-methyl-4phthalimidobutylamino)quinoline as a yellow crystalline solid $(3.3 \mathrm{~g}) \cdot{ }^{1} \mathrm{H}$ NMR $\delta\left(\mathrm{CDCl}_{3}\right): 1.27(3 \mathrm{H}, \mathrm{d}, \mathrm{J}=6.0 \mathrm{~Hz})$, 1.60-1.90 (4H, m), $3.62(1 \mathrm{H}, \mathrm{m}), 3.73(2 \mathrm{H}, \mathrm{t}, \mathrm{J}=6.8 \mathrm{~Hz}), 3.84$ $(3 \mathrm{H}, \mathrm{s}), 5.46(2 \mathrm{H}, \mathrm{s}), 5.54(1 \mathrm{H}, \mathrm{d}, \mathrm{J}=6.8 \mathrm{~Hz}), 6.31(2 \mathrm{H}, \mathrm{s})$, $6.91(1 \mathrm{H}, \mathrm{d}, \mathrm{J}=8.8 \mathrm{~Hz}), 7.23(1 \mathrm{H}, \mathrm{t}, \mathrm{J}=7.6 \mathrm{~Hz}), 7.38(2 \mathrm{H}, \mathrm{t}$, $7.6 \mathrm{~Hz}), 7.51(2 \mathrm{H}, \mathrm{d}, \mathrm{J}=7.6 \mathrm{~Hz}), 7.66(2 \mathrm{H}, \mathrm{m}) 7.78(2 \mathrm{H}, \mathrm{m})$, $7.81(1 \mathrm{H}, \mathrm{d}, 8.8 \mathrm{~Hz})$; HRESIMS $[\mathrm{M}+\mathrm{H}]^{+} \mathrm{m} / z 496.2232$ (calculated for $\left(\mathrm{C}_{30} \mathrm{H}_{29} \mathrm{~N}_{3} \mathrm{O}_{4}+\mathrm{H}\right)^{+}$496.2236).

\section{2-hydroxyprimaquine diphosphate}

A mixture of 2-benzyloxy-6-methoxy-8-(1-methyl-4phthalimidobutylamino)quinoline (2.0 g) and hydrazine hydrate $(1.5 \mathrm{ml})$ in ethanol $(50 \mathrm{ml})$ was refluxed for four hours. The reaction mixture was cooled and the white precipitate was separated by filtration. The filtrate was evaporated and the gummy residue was dissolved in $\mathrm{CH}_{2} \mathrm{Cl}_{2}$ and washed $(\times 2)$ aqueous potassium hydroxide (10\%), water, dried over $\mathrm{Na}_{2} \mathrm{SO}_{4}$ and evaporated to give $N^{4}$-(2-(benzyloxy)-6-methoxyquinolin-8-yl)pentane1,4-diamine. This product was dissolved in ethanol $(50 \mathrm{ml})$ and refluxed with hydrazine hydrate $(2 \mathrm{ml})$ and Pd/C (10\%, $200 \mathrm{mg})$ for two hours. The catalyst was removed by filtration and the filtrate was evaporated under reduced pressure. The resulting yellow solid was dissolved in ethanol $(10 \mathrm{ml})$ and $\mathrm{H}_{3} \mathrm{PO}_{4}(85 \%, 2 \mathrm{ml})$ was added drop-wise under stirring. The supernatant was removed by decantation and the gummy deposit was washed with ethanol and crystallized from water ethanol to give 2-OH-PQ diphosphate (1.6 g). ${ }^{1} \mathrm{H}$ NMR $\delta$ $\left(\mathrm{CDCl}_{3}\right): 0.93(3 \mathrm{H}, \mathrm{d}, \mathrm{J}=6.0 \mathrm{~Hz}), 1.28(1 \mathrm{H}, \mathrm{m}), 1.40$ $(1 \mathrm{H}, \mathrm{m}) 1.42-1.58(2 \mathrm{H}, \mathrm{m}), 2.77(2 \mathrm{H}, \mathrm{t}, \mathrm{J}=7.6 \mathrm{~Hz})$, $3.08(1 \mathrm{H}, \mathrm{m}), 3.44(3 \mathrm{H}, \mathrm{s}), 5.76(1 \mathrm{H}, \mathrm{brs}), 5.84(1 \mathrm{H}, \mathrm{brs})$, $6.06(\mathrm{H}, \mathrm{d}, \mathrm{J}=9.2 \mathrm{~Hz}), 7.12(1 \mathrm{H}, \mathrm{d}, 9.2 \mathrm{~Hz})$; HRESIMS $[\mathrm{M}+\mathrm{H}]^{+} m / z 276.1710$ (calculated for $\left(\mathrm{C}_{15} \mathrm{H}_{21} \mathrm{~N}_{3} \mathrm{O}_{2}+\mathrm{H}\right)^{+}$ 276.1712).

\section{Synthesis of 3-hydroxyprimaquine diphosphate 3-hydroxy-6-methoxy-8-nitroquinoline}

A mixture of 3-bromo-6-methoxy-8-nitroquinoline [32], (2 g, $7 \mathrm{~mm}), \mathrm{Pd}_{2} \mathrm{dba}_{3}(130 \mathrm{mg}, 0.14 \mathrm{~mm}), 2$-di-tertbutylphosphino-2',4',6' -triisopropyibiphenyl (140 mg, $0.33 \mathrm{~mm})$, and $\mathrm{KOH}(1.2 \mathrm{~g}, 21 \mathrm{~mm})$ in water:dioxane $(1: 1,20 \mathrm{ml})$ was heated at $95^{\circ} \mathrm{C}$ for two hours under nitrogen atmosphere. The reaction mixture was poured onto ice, acidified with hydrochloric acid and filtered. The solid obtained was purified by column chromatography on silica gel with hexanes:ethyl acetate 7:3 as the eluent to give a yellow crystalline solid $(1.36 \mathrm{~g}) .{ }^{1} \mathrm{H}$ NMR $\delta\left(\mathrm{CDCl}_{3} / \mathrm{CD}_{3} \mathrm{OD}\right): 3.79(3 \mathrm{H}, \mathrm{s}), 7.01(1 \mathrm{H}, \mathrm{d}, \mathrm{J}=2.8 \mathrm{~Hz})$, $7.26(1 \mathrm{H}, \mathrm{d}, \mathrm{J}=2.8 \mathrm{~Hz}) 7.35(\mathrm{H}, \mathrm{d}, \mathrm{J}=2.4 \mathrm{~Hz}), 8.35(1 \mathrm{H}, \mathrm{d}$, 2.4 Hz); HRESIMS $[\mathrm{M}+\mathrm{H}]^{+} \mathrm{m} / z 221.0572$ (calculated for $\left(\mathrm{C}_{10} \mathrm{H}_{9} \mathrm{~N}_{2} \mathrm{O}_{4}+\mathrm{H}\right)^{+}$221.0562).

\section{3-benzyloxy-6-methoxy-8-nitroquinoline}

A mixture of 3-hydroxy-6-methoxy-8-nitroquinoline (1.35 g, 6.1.mm), benzyl bromide (1.71 g, $10 \mathrm{~mm})$, and $\mathrm{Cs}_{2} \mathrm{CO}_{3}$ $(3.25 \mathrm{gm}, 10 \mathrm{~mm})$ in DMF $(15 \mathrm{ml})$ was stirred at $65^{\circ} \mathrm{C}$ for six hours. The reaction mixture was poured into cold water and filtered. The crude product was chromatographed over silica gel and elution with hexanes:ethyl acetate 9:1 gave 3-benzyloxy-6-methoxy-8-nitroquinoline (1.8 gm). ${ }^{1} \mathrm{H}$ NMR $\delta\left(\mathrm{CDCl}_{3}\right): 3.81(3 \mathrm{H}, \mathrm{s}), 5.35(2 \mathrm{H}, \mathrm{s}), 6.49(1 \mathrm{H}, \mathrm{d}$, $\mathrm{J}=2.4 \mathrm{~Hz}), 6.68(1 \mathrm{H}, \mathrm{d}, \mathrm{J}=2.4 \mathrm{~Hz}) 7.28(1 \mathrm{H}, \mathrm{t}, \mathrm{J}=6.8 \mathrm{~Hz})$, $7.35(2 \mathrm{H}, \mathrm{t}, \mathrm{J}=6.8 \mathrm{~Hz}), 7.48(2 \mathrm{H}, \mathrm{d}, \mathrm{J}=6.8 \mathrm{~Hz}), 8.09(1 \mathrm{H}$, d, $2.4 \mathrm{~Hz}), 8.75(1 \mathrm{H}, \mathrm{d}, 2.4 \mathrm{~Hz})$; HRESIMS $[\mathrm{M}+\mathrm{H}]^{+} \mathrm{m} / \mathrm{z}$ 311.1050 (calculated for $\left(\mathrm{C}_{17} \mathrm{H}_{15} \mathrm{~N}_{2} \mathrm{O}_{4}+\mathrm{H}\right)^{+} 311.1032$ ). 


\section{3-benzyloxy-6-methoxy-8-(1-methyl-4-phthalimidobutylamino) quinoline}

A mixture of 3-benzyloxy-6-methoxy-8-nitroquinoline (1.8 gm), Raney-Ni (1 g) and hydrazine hydrate $(2 \mathrm{ml})$ in ethanol $(30 \mathrm{ml})$ was refluxed for four hours. The catalyst was removed by filtration through a celite plug and the filtrate was evaporated under reduced pressure. The gummy residue was partitioned between water and $\mathrm{CH}_{2} \mathrm{Cl}_{2}$ and the organic layer was dried and evaporated to yield 8-amino-3benzyloxy-6-methoxyquinoline (1.6 g, $5.7 \mathrm{~mm})$. This product and 2-oxo-5-phthalimido pentane (1.5 g, $6.8 \mathrm{~mm})$ were coupled in glacial acetic acid $(15 \mathrm{ml})$ as described above for 2-benzyloxy analog to afford 3-benzyloxy-6-methoxy-8-(1methyl-4-phthalimidobutylamino)quinoline as a yellow crystalline solid $(1.9 \mathrm{~g}) .{ }^{1} \mathrm{H}$ NMR $\delta\left(\mathrm{CDCl}_{3}\right): 1.27(3 \mathrm{H}, \mathrm{d}$, $\mathrm{J}=6.0 \mathrm{~Hz}), 1.60-1.90(4 \mathrm{H}, \mathrm{m}), 3.64(1 \mathrm{H}, \mathrm{m}), 3.72(2 \mathrm{H} \mathrm{t}$, $\mathrm{J}=7.6 \mathrm{~Hz}), 3.86(3 \mathrm{H}, \mathrm{s}), 5.15(2 \mathrm{H}, \mathrm{s}), 5.87(1 \mathrm{H}, \mathrm{d}, \mathrm{J}=$ $8.4 \mathrm{~Hz}), 6.15(1 \mathrm{H}, \mathrm{d}, \mathrm{J}=2.4 \mathrm{~Hz}), 6.22(1 \mathrm{H}, \mathrm{d}, \mathrm{J}=2.4 \mathrm{~Hz})$, $7.26(1 \mathrm{H}, \mathrm{d}, \mathrm{J}=2.4 \mathrm{~Hz}), 7.35(1 \mathrm{H}, \mathrm{t}, \mathrm{J}=6.8 \mathrm{~Hz}), 7.41(2 \mathrm{H}, \mathrm{t}$, $7.2 \mathrm{~Hz}), 7.47$ (2H, d, J = 7.2 Hz), $7.68(2 \mathrm{H}, \mathrm{m}) 7.81(2 \mathrm{H}, \mathrm{m})$, $8.33(1 \mathrm{H}, \mathrm{d}, 2.4 \mathrm{~Hz})$; HRESIMS $[\mathrm{M}+\mathrm{H}]^{+} \mathrm{m} / z 496.2229$ (calculated for $\left(\mathrm{C}_{30} \mathrm{H}_{29} \mathrm{~N}_{3} \mathrm{O}_{4}+\mathrm{H}\right)^{+}$496.2236).

\section{3-hydroxyprimaquine diphosphate}

The phthalimide protecting group of 3-benzyloxy-6methoxy-8-(1-methyl-4-phthalimidobutylamino)quinoline $(1.8 \mathrm{~g})$ was removed by reacting with hydrazine hydrate $(1 \mathrm{ml})$ in ethanol $(40 \mathrm{ml})$ as described for 2-benzyloxy analog to give $N^{4}$-(3-(benzyloxy)-6-methoxyquinolin-8-yl) pentane-1,4-diamine as a yellow gum. The benzyl group was removed as described above by refluxing for two hours in ethanol $(30 \mathrm{ml})$ in the presence of hydrazine hydrate $(1 \mathrm{ml})$ and $\mathrm{Pd} / \mathrm{C}(10 \%, 200 \mathrm{mg})$. The reaction was worked up and 3-OH-PQ was crystallized as diphosphate $(1.4 \mathrm{~g}) .{ }^{1} \mathrm{H}$ NMR $\delta\left(\mathrm{CDCl}_{3}\right): 1.10(3 \mathrm{H}, \mathrm{d}, \mathrm{J}=6.0 \mathrm{~Hz}), 1.30-$ $1.65(4 \mathrm{H}, \mathrm{m}), 2.81(2 \mathrm{H}, \mathrm{t}, \mathrm{J}=7.2 \mathrm{~Hz}), 3.45(1 \mathrm{H}, \mathrm{m}), 3.65$ $(3 \mathrm{H}, \mathrm{s}), 6.28(1 \mathrm{H}, \mathrm{brs}), 7.21(\mathrm{H}, \mathrm{d}, \mathrm{J}=2.4 \mathrm{~Hz}), 7.97(1 \mathrm{H}, \mathrm{d}$, 2.4 Hz); HRESIMS $[\mathrm{M}+\mathrm{H}]^{+} \mathrm{m} / z 276.1728$ (calculated for $\left(\mathrm{C}_{15} \mathrm{H}_{21} \mathrm{~N}_{3} \mathrm{O}_{2}+\mathrm{H}\right)^{+}$276.1712).

\section{Other chemicals and reagents}

Nicotinamide adenine dinucleotide phosphate, reduced form (NADPH), glucose-6-phosphate (G6P), G6PD and magnesium chloride $\left(\mathrm{MgCl}_{2}\right)$ were purchased from Sigma-Aldrich (St Louis, MO, USA). HPLC-grade acetonitrile and methanol were purchased from Fisher Scientific (Fair Lawn, NJ, USA). Water for the HPLC mobile phase was purified in a Milli-Q system (Millipore, Bedford, MA, USA). Baculovirus-insect cell expressed recombinant cytochrome-P450 supersomes containing CYP2D6 (1 nmole CYP per $\mathrm{mL}$ ) were purchased from (BD Biosciences, Billerica, MA, USA) and stored at $-80^{\circ} \mathrm{C}$ until used.

\section{Primaquine/CYP2D6 incubation}

The in vitro primaquine metabolism reactions were set up in a clear 96-well plate. Thawed suspensions of the supersomes were diluted with potassium phosphate buffer (50 mM; $\mathrm{pH}=7.4)$ and aliquots were dispensed in a clear 96-well plate. Primaquine (racemate or the appropriate enantiomer, 50:50 mixture of ${ }^{12} \mathrm{C}$ and ${ }^{13} \mathrm{C}$-labeled) was added and the mixture pre-incubated at $37^{\circ} \mathrm{C}$ for $10 \mathrm{~min}$. Metabolic reactions were initiated by adding the NADPH-regenerating solution containing magnesium chloride, G6P and G6PD. The final components of the incubations were: reduced NADP (1 mM), G6P (5 mM), $\mathrm{MgCl}_{2}$ (5 mM), G6PD (1 U/mL), recombinant CYP2D6 (0.5 $\mathrm{mg} / \mathrm{mL})$ and primaquine (varying concentrations). Metabolic reactions were terminated at predetermined time-points through the addition of equal volume of icecold methanol containing $0.5 \mu \mathrm{g} / \mathrm{mL} 6-\mathrm{D}_{3}$-methoxyprimaquine as internal standard. The mixtures were kept on ice for one hour and then centrifuged (14,000 rpm, -4 degree centigrade, $20 \mathrm{~min})$. Clear supernatants were kept for LC-MS analysis. All incubations were performed in duplicate for intra-day agreement and repeated on separate days for inter-day comparisons. Control incubations included: a) those with primaquine but without the supersomes; b) those with supersomes and primaquine but without the start solution; and, c) those without the primaquine. There was no organic solvent in the incubation mixtures as the contents were water-soluble. After the initial determination of the kinetic parameters of primaquine and its enantiomers, the probe primaquine concentrations for the metabolite identification and quantification were less than the determined $K m$ value.

Detection, identification and quantification of metabolites Liquid chromatography - mass spectrometry (LC-MS) method for simultaneous analysis of primaquine and its metabolites as reported earlier was employed in this study [9]. Total separation and elution of the analytes were achieved within $10 \mathrm{~min}$ retention time, using the ACQUITY UHPLC ${ }^{\mathrm{m}}$, BEH Shield RP18 column (100 mm $\times$ $2.1 \mathrm{~mm}$ I.D., $1.7 \mathrm{~mm}$ ) equipped with an LC-18 guard column (Vanguard $2.1 \times 5 \mathrm{~mm}$, Waters Corp, Milford, MA, USA) on an ACQUITY UHPLC system (Waters Corp, Milford, MA, USA) to which a conditioned auto-sampler (at $20^{\circ}$ $\mathrm{C})$ was attached. The mobile phase, consisting of water with $0.05 \%$ formic acid (A) and acetonitrile with $0.05 \%$ formic acid (B), was applied at a flow rate of $0.25 \mathrm{ml} / \mathrm{min}$ in the following linear gradient elution: $0 \mathrm{~min}, 90 \% \mathrm{~A}: 10 \% \mathrm{~B}$ in next 5 min to $63 \% \mathrm{~A}: 37 \% \mathrm{~B}$, then for $3 \mathrm{~min} 37 \% \mathrm{~A}: 63 \% \mathrm{~B}$ and to $100 \% \mathrm{~B}$ in next $2 \mathrm{~min}$. Each run was followed by a 3-min wash with $100 \% \mathrm{~B}$ and an equilibration period of $3.5 \mathrm{~min}$ with $90 \% \mathrm{~A} / 10 \% \mathrm{~B}$. Ten $\mu \mathrm{L}$ of each sample were injected, and peaks assigned with respect to the mass of the compounds and comparison of the retention times. 
Metabolites in the accurate mass data were found using the Metabolynx ${ }^{\oplus}$ software. The data were searched using predicted metabolite mass, mass defects, isotope, and fragmentation patterns. Each sample was subjected to data acquisition in full scan and data-dependent positive MS/MS, targeted MS/MS (ESI positive ionization mode) and high-resolution MS (HRMS) modes using the Waters ACQUITY ${ }^{\mathrm{TM}}$ XEVO QTOF Mass Spectrometer (Waters Corporation, Manchester, UK) connected to the UHPLC system via an electrospray ionization (ESI) interface. Identification of each metabolite was assisted by its HRMS data, which were used to calculate their elemental compositions. The full scan mass data were screened and filtered using Waters MetaboLynx XS software. The qualitative metabolite identification was performed using this software package.

\section{Data analysis}

After suitable calibrations of the substrates and the synthetic metabolites, the initial rate of primaquine (racemate and its enantiomers) metabolism was profiled against the concentration incubated using the SigmaPlot Enzyme Kinetic Software, Module 13.0 (Systat Software Inc, Chicago, IL, USA). The pattern of metabolism was characterized through the Michaelis-Menten plot $(\mathrm{N}=4$; S.D. $<0.05$ in all cases, $22 \geq 0.9$ ) from which the kinetic parameters were determined. Identified metabolites were quantified and profiled against time.

\section{Results}

\section{Comparative kinetics for metabolism of primaquine enantiomers}

Varying concentrations of primaquine and its enantiomers were subjected to human CYP2D6-catalyzed metabolism. The initial velocity of metabolism $\left(V_{o}\right)$ was profiled against substrate concentration yielding a Michaelis-Menten-type curve (Figure 1A). The rate of metabolism of (+)-primaquine was significantly higher $(50 \%$ depletion of $20 \mathrm{mM}$ primaquine in $120 \mathrm{~min}$ ) compared to (-)-primaquine (30\% depletion). The rate of metabolism of racemic primaquine was similar to (-)-primaquine. The estimated Vmax $(\mu \mathrm{mol} / \mathrm{min} / \mathrm{mg})$ are $0.87,0.75$ and 0.42 for $( \pm)$-primaquine, (+)-primaquine and (-)-primaquine, respectively. The estimated Michaelis-Menten constants $\left(K_{m}\right)(\mu \mathrm{M})$ for $( \pm)$-primaquine, $(+)$-primaquine and $(-)$-primaquine were 24.2, 33.1 and 21.6, respectively. The results in Figure 1A illustrate that (-)-primaquine saturated CYP2D6 earlier than (+)-primaquine or the racemic mixture. Additionally, the Michaelis-Menten kinetic analyses indicated that (-)-primaquine has a higher affinity for CYP2D6 as compared to $(+)$-primaquine.

The depletion of primaquine substrates from the initial concentration $\left(C_{o}\right)$ vs time is presented in Figure 1B. After two hours, the amount of (+)-primaquine depleted is more than $50 \%$ greater than the $(-)$-primaquine consumed. These profiles suggest further that the CYP2D6 metabolism of primaquine is stereo-selective and more favourable towards $(+)$-primaquine.

\section{Metabolite identification}

Following the incubation of primaquine (racemate and individual enantiomers) in vitro with recombinant human CYP2D6, several metabolites were identified. Quantitatively these metabolites were formed at varying levels with $(+)$ and $(-)$-primaquine. Table 1 provides a list of the identified metabolites and their comparative abundance in the enantiomers. Formation of some of these metabolites has been reported with the racemic primaquine [18]. Three stable mono-hydroxylated metabolites namely, 2-OH-PQ, 3-OH-PQ and 4-OH-PQ were identified, confirmed with analytical standards and quantified. 5,6-orthoquinone analog $(m / z 260)$, the product of the spontaneous transformation of 5-OH-PQ was identified, as one of the most abundant primaquine metabolite formed with CYP2D6. Smaller amounts of two other metabolites corresponding to dihydroxylated metabolites
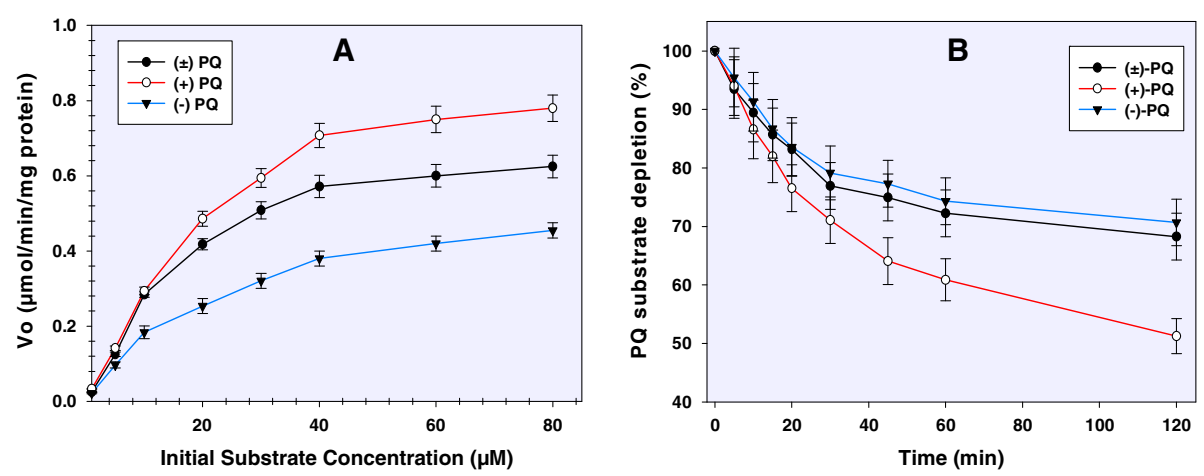

Figure 1 Concentration- and time-dependent metabolism of primaquine and its enantiomers. (A) Steady state kinetics of primaquine and its enantiomers - a profile of the initial rate of substrate depletion $\left(V_{0}\right)$ against concentration and $(\mathbf{B})$ comparative depletion of primaquine and its enantiomers from human CYP2D6 incubation over 2 hr. Each point represents mean values \pm S.D. $(n=4)$, 
Table 1 Identified metabolites from CYP2D6-mediated metabolism of primaquine and its enantiomers

\begin{tabular}{|c|c|c|c|}
\hline Peak $[\mathrm{M}+\mathrm{H}]^{+}$ & RT & Formula & Remarks \\
\hline 260.13 & 1.5 & $\mathrm{C}_{14} \mathrm{H}_{17} \mathrm{~N}_{3} \mathrm{O}_{2}$ & $\begin{array}{l}\text { Oxidized product of 5-OH-PQ, preferentially generated from the (+)-primaquine (twice as much } \\
\text { compared to (-)-primaquine) }\end{array}$ \\
\hline 261.16 & 8.7 & $\mathrm{C}_{15} \mathrm{H}_{20} \mathrm{~N}_{2} \mathrm{O}_{2}$ & Oxidative deamination of primaquine and subsequent reduction to primaquine alcohol. \\
\hline 275.14 & 8.8 & $\mathrm{C}_{15} \mathrm{H}_{18} \mathrm{~N}_{2} \mathrm{O}_{3}$ & $\begin{array}{l}\text { Possibly terminal amine oxidation, with oxidation and quinone-imine formation on quinoline } \\
\text { ring, predominantly formed from (-)-primaquine (2:1) }\end{array}$ \\
\hline 276.17 & 2.08 & $\mathrm{C}_{15} \mathrm{H}_{21} \mathrm{~N}_{3} \mathrm{O}_{2}$ & $\begin{array}{l}\text { Identified as 4-OH-PQ; Formed } 5 \text { times more predominantly with (-)-primaquine than } \\
(+) \text {-primaquine }\end{array}$ \\
\hline 276.17 & 3.64 & $\mathrm{C}_{15} \mathrm{H}_{21} \mathrm{~N}_{3} \mathrm{O}_{2}$ & Identified as 2-OH-PQ; Generated in the ratio 4:1 by (+)-primaquine vs (-)-primaquine \\
\hline 276.17 & 4.78 & $\mathrm{C}_{15} \mathrm{H}_{21} \mathrm{~N}_{3} \mathrm{O}_{2}$ & Identified as 3-OH-PQ; Generated in the ratio 2:3 by (+)-primaquine and (-)-primaquine \\
\hline 290.15 & 3.42 & $\mathrm{C}_{15} \mathrm{H}_{20} \mathrm{~N}_{3} \mathrm{O}_{3}$ & $\begin{array}{l}\text { Putatively identified as dihydroxylated primaquine metabolite converted to quinone-imines, } \\
\text { generated in the ratio } 3: 2 \text { by }(-) \text {-primaquine and (+)-primaquine. }\end{array}$ \\
\hline 290.15 & 4.56 & $\mathrm{C}_{15} \mathrm{H}_{20} \mathrm{~N}_{3} \mathrm{O}_{3}$ & $\begin{array}{l}\text { Putatively identified as a dihydroxylated primaquine metabolite converted to quinone-imine; } \\
\text { Generated more predominantly with (-)-primaquine, minimally with }( \pm) \text {-primaquine and not } \\
\text { detected with (+)-primaquine }\end{array}$ \\
\hline 292.16 & 4.59 & $\mathrm{C}_{15} \mathrm{H}_{21} \mathrm{~N}_{3} \mathrm{O}_{3}$ & Putatively identified as dihydroxyprimaquine; Generated with (-)-primaquine only \\
\hline 306.14 & 3.65 & $\mathrm{C}_{15} \mathrm{H}_{20} \mathrm{~N}_{3} \mathrm{O}_{4}$ & $\begin{array}{l}\text { Putatively identified as trihydroxylated primaquine converted to the quinone-imine; selectively } \\
\text { generated with (+)-primaquine }\end{array}$ \\
\hline 308.16 & 1.4 & $\mathrm{C}_{15} \mathrm{H}_{21} \mathrm{~N}_{3} \mathrm{O}_{4}$ & $\begin{array}{l}\text { Putatively identified as trihydroxyprimaquine; detected in trace amount; more prominently } \\
\text { generated with (+)-primaquine }\end{array}$ \\
\hline
\end{tabular}

and identified as respective quinone-imines or orthoquiones $(\mathrm{m} / \mathrm{z} 290)$ were also identified. The two dihydroxymetabolites exhibited different retention times (3.42 and $4.5 \mathrm{~min}$ ), probably due to hydroxylation at different positions on the quinoline ring. Trace amounts of primaquine terminal alcohol were also detected. Figure 2 shows suggested pathways for metabolism of primaquine by human CYP2D6.

\section{2-hydroxyprimaquine}

The identification of 2-OH-PQ was confirmed through the availability of its synthetic standard. 2-OH-PQ was preferentially formed from (+)-primaquine peaking early within $15 \mathrm{~min}$ at about $390 \mathrm{ng} / \mathrm{mL}$ compared to the peak of less than $100 \mathrm{ng} / \mathrm{mL}$ generated by (-)-primaquine (Figure 3A). The rate of metabolism of the racemic $( \pm)$-primaquine to 2-OH-PQ was similar to that of the (+)-primaquine. A small reduction in the level of 2-OHPQ in was recorded for (+)-primaquine after $30 \mathrm{~min}$, with the level of 2-OH-PQ levelling at about $300 \mathrm{ng} / \mathrm{mL}$, presumably reflecting further metabolism of this species. However, the level of 2-OH-PQ generated from (-)-primaquine peaked at $15 \mathrm{~min}$. The pattern of 2 $\mathrm{OH}-\mathrm{PQ}$ generation suggests that the amount observed with the racemic $( \pm)$-primaquine at two hours approximates the cumulative sum of those generated by the individual enantiomers. Thus, the enantiomers do not compete with each other for their metabolism to 2-OH-PQ with CYP2D6. The 2-OH-PQ accounted for about 15-17\% of total CYP2D6-mediated metabolism of $(+)$-primaquine or $( \pm)$-primaquine.

\section{3-hydroxyprimaquine}

With a retention time of $3.6 \mathrm{~min}$, synthetic $3-\mathrm{OH}-\mathrm{PQ}$ was used to confirm the formation of this metabolite with CYP2D6. The level of 3-OH-PQ peaked at $30 \mathrm{~min}$ and was formed with 1.5-fold more abundance from $(-)$-primaquine than with $(+)$-primaquine, while the levels of 3-OH-PQ generated from $( \pm)$-primaquine were apparently the cumulative generation from the individual enantiomers. The 3-OH-PQ also was identified as a stable metabolite (Figure $3 \mathrm{~B}$ ).

\section{4-hydroxyprimaquine}

The identity of 4-OH-PQ was also confirmed through the synthetic standard with a chromatographic retention time of $2.1 \mathrm{~min}$ and $\mathrm{m} / \mathrm{z} 276.25$. It was preferentially formed with (-)-primaquine, generating five times the quantity observed with (+)-primaquine. It accounts for about $22 \%$ of the total metabolism of (-)-primaquine. The pattern of its generation appears to be complementary to the generation of 2-OH-PQ. It peaked at $30 \mathrm{~min}$ in (-)-primaquine with slight drop in the quantity generated over the next 90 min (Figure 3C).

\section{5-hydroxyprimaquine}

5,6-orthoquinone analog $(m / z 260)$, the product of the spontaneous transformation of 5-OH-PQ was identified based on its retention time (1.65 $\mathrm{min})$ and fragmentation pattern. It was semi-quantified relative to the parent primaquine calibration. The LC-MS/MS profile of this product as identified in primaquine and CYP2D6 reaction 


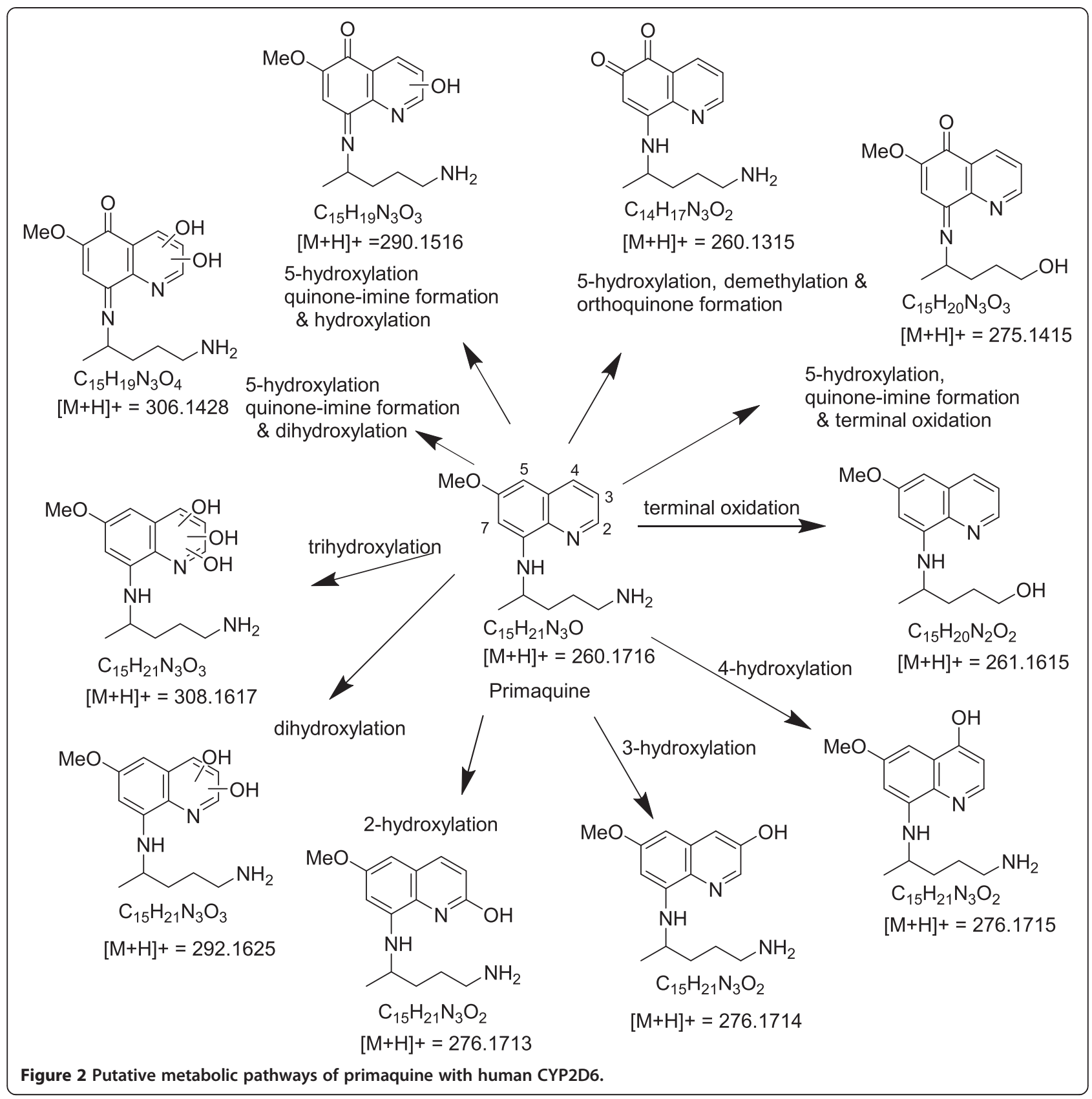

mixture was identical to the oxidation products formed with the synthetic 5-OH-PQ. Based on semi-quantification of this 5,6-orthoquinone product as a marker for 5-OH$\mathrm{PQ}$, the generation of $5-\mathrm{OH}-\mathrm{PQ}$ is estimated to account for $18-20 \%$ of the CYP2D6-mediated conversion of (+)-primaquine. The formation of this 5-OH-PQ product from (+)-primaquine was more than twice as abundant as with (-)-primaquine. However, generation of 5-OH-PQ marker product from $( \pm)$ primaquine was of about half the amount from (-)-primaquine and more than four-fold less than that generated from (+)-primaquine. This observation suggested that at these concentrations, the individual primaquine enantiomers may be competing with each other for metabolism of primaquine through this pathway (Figure 3D).

\section{Dihydroxylated primaquine metabolite converted to quinone-imines}

The two metabolites, corresponding to dihydroxylated primaquine presumably degraded or further metabolized to quinone-imines or orthoquinones $(\mathrm{m} / z 290)$, were identified in small quantities. Generation of these metabolites also exhibited differential pattern with racemate primaquine and individual enantiomers (Figure 4). The 

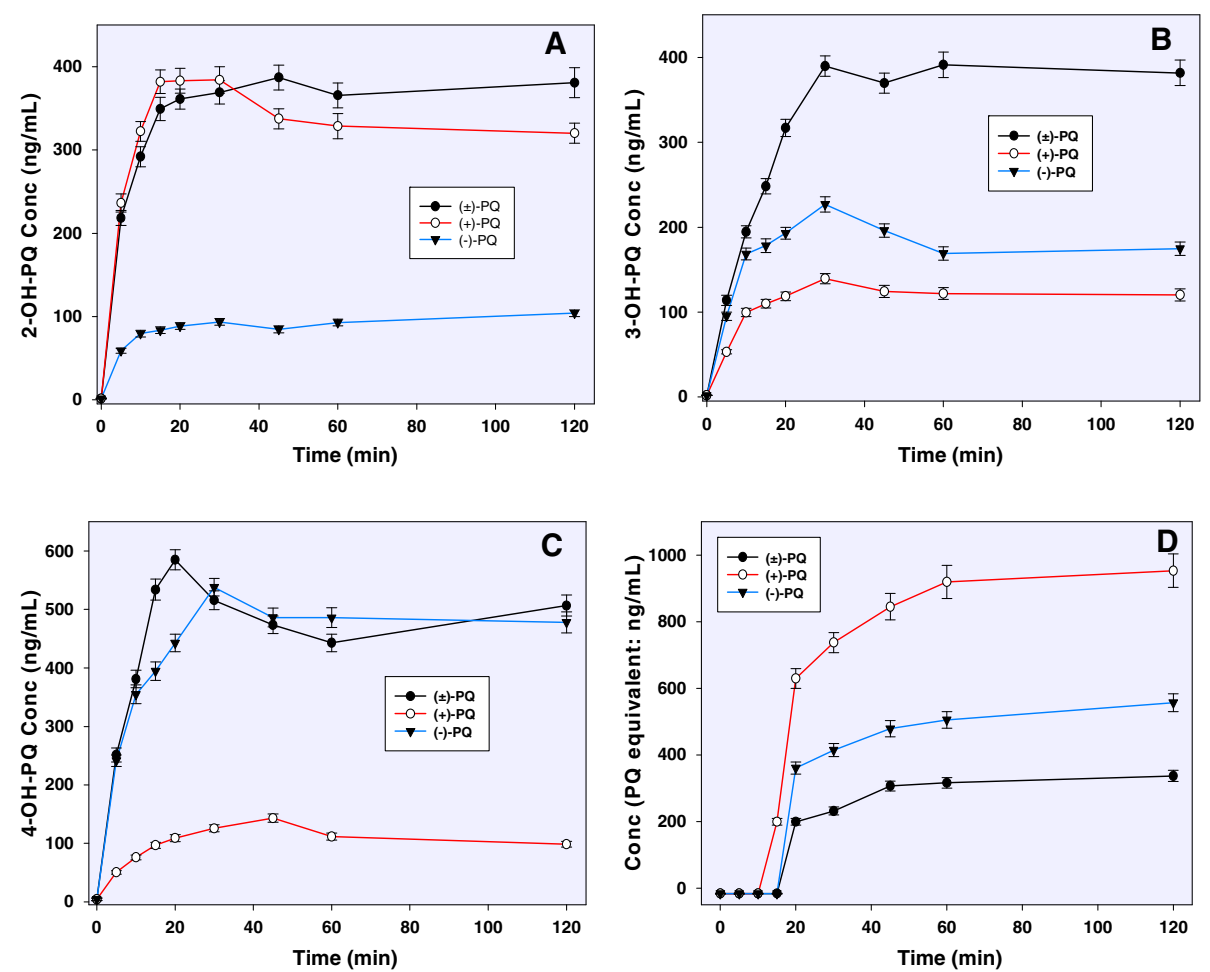

Figure 3 Comparative kinetics of formation of (A) 2-hydroxyprimaquine; (B) 3-hydroxyprimaquine; (C) 4-hydroxyprimaquine and (D) 5-hydroxyprimaquine (expressed as the orthoquinone, relative to primaquine calibration) on metabolism of primaquine (racemate and individual enantiomers) by human CYP2D6. Each point represents mean value \pm S.D. $(n=4)$.

differential positions of $\mathrm{OH}$ on the quinoline ring generated the metabolites with distinctly different retention time. The first of these two quinone-imine products appeared at $3.4 \mathrm{~min}$ and was generated in the ratio $3: 2$, $(-)$-primaquine $v s(+)$-primaquine (Figure 4A). It was generated in higher levels from the individual enantiomers than from the racemic primaquine, although the level of this metabolite was very low based on semiquantitation. The second quinone-imine appeared at $4.56 \mathrm{~min}$ and was almost exclusively generated from the (-)-primaquine. Only a small amount of this metabolite appeared transiently between 10 and $30 \mathrm{~min}$ from $( \pm)$-primaquine. No formation of this metabolite was detected from $(+)$-primaquine. (Figure 4B).

\section{Dihydroxyprimaquine}

Formation of a metabolite, predicted as dihydroxyprimaquine $(m / z 292)$ and presumably formed by further hydroxylation of a monohydroxy primaquine, was detected in significant levels (Figure 5A). The exact hydroxylation
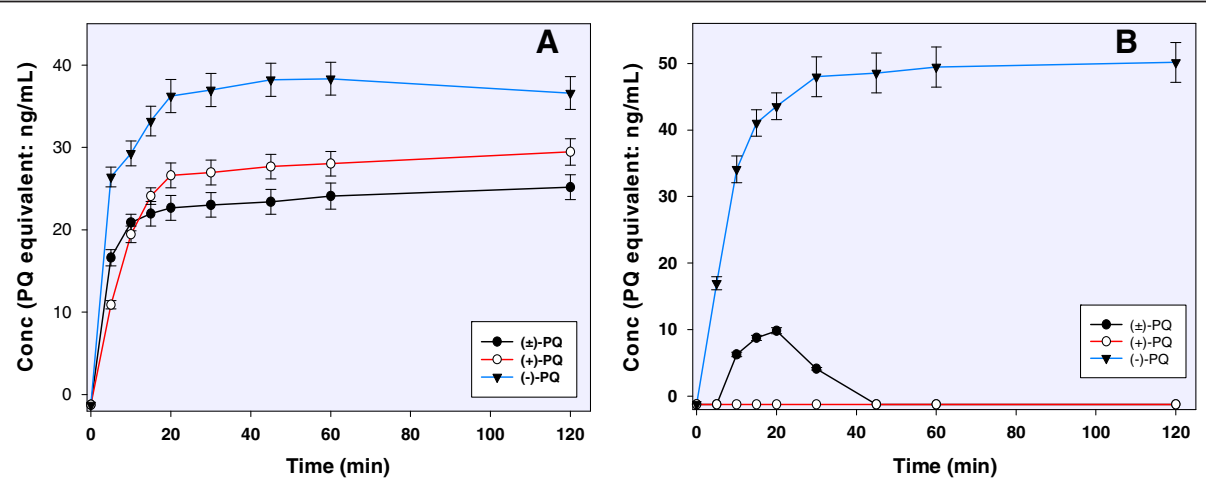

Figure 4 Semi-quantitation of the human CYP2D6-catalyzed enantio-selective generation of quinone-imine derivative of dihydroxylated primaquine metabolites (A) RT $3.42 \mathrm{~min}$ metabolite and (B) RT 4.56 metabolite, identified as quinone-imines ( $\mathrm{m} / \mathrm{z} 290)$ degradation product. Each point shows mean value \pm S.D. $(n=4)$. 

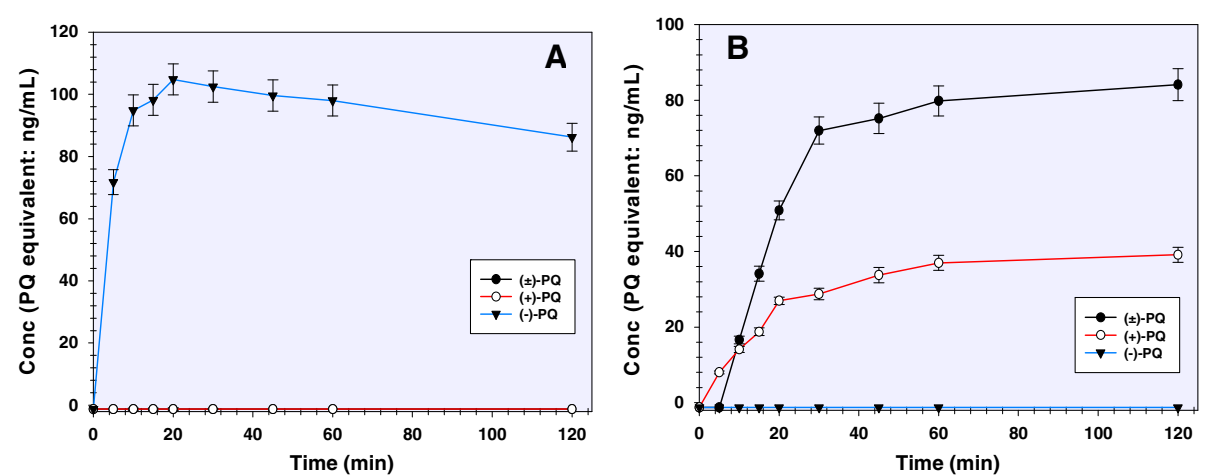

Figure 5 The human CYP2D6-catalyzed enantio-selective metabolism of primaquine showing the generation of (A) dihydroxyprimaquine (semi-quantified in relation to primaquine calibration) and (B) primaquine alcohol. Each point shows mean value \pm S.D. $(n=4)$.

site in this metabolite could not be established. This metabolite, which was exclusively generated from (-)-primaquine, peaked within $20 \mathrm{~min}$ incubation and remained high until end of $120 \mathrm{~min}$ of incubation. This metabolite was not detected in the CYP2D6 incubation mixtures with $(+)$-primaquine or $( \pm)$-primaquine.

\section{Primaquine alcohol}

Formation of primaquine alcohol has also been reported earlier $[9,18]$. The identity of this metabolite was confirmed based on the similarity of its MS/MS fragmentation with the synthetic primaquine alcohol, having the same retention time and UV spectrum. Primaquine alcohol was exclusively generated from the $(+)$-primaquine enantiomer (Figure 5B).

\section{Discussion}

The results presented in this paper confirm earlier reports regarding generation of multiple mono-hydroxylated metabolites of primaquine in vitro by primary human hepatocytes [16,17] and CYP2D6 [18,19]. However, previous studies [16-19] did not provide evidence regarding the specific sites of oxidation on the quinoline ring. The results presented here provide definitive evidence that CYP2D6-mediated oxidation of primaquine occurred at several different positions on the quinoline ring. The identification and quantification of three mono-hydroxylated primaquine metabolites were successfully carried out. Mono-hydroxylated primaquine metabolites were identified as 2-, 3- and 4-OH-PQ by comparing the retention times on LC and MS/MS fragmentation data with those of the synthetic standards. An orthoquinone product of a fourth hydroxyl product was identified as a major CYP2D6 metabolite and is a likely marker for the 5 hydroxylation pathway. Definitive generation of these metabolites from ${ }^{13} \mathrm{C}(6)$-primaquine $/{ }^{12} \mathrm{C}$-primaquine (1:1) was confirmed by identification of twin mass peaks for each metabolite. Even though both 2- and 4-OH-PQ can form oxidized products with keto-imine groups, they appear to be stable due to quinolinol-quinolone tautomerism. Further, analysis of the data showed significant quantitative differences in generation of these metabolites from the individual primaquine enantiomers.

This is the first report on differential preferences of human CYP2D6 for individual primaquine enantiomers. The rate of metabolism of (+)-primaquine was about 2 fold higher compared to (-)-primaquine. However, the rate of metabolism of racemic primaquine was similar to (-)-primaquine. This may be due to possible inhibition of the metabolism of one enantiomer by the other. Supposedly, (+)- and (-)-primaquine produce respective (+)and (-)-metabolites. No isomerization has been detected with PQ and carboxy PQ and has been earlier confirmed with carboxyprimaquine generated in vitro with human hepatocytes (9).

Relative importance of individual hydroxylated primaquine metabolites in efficacy $v s$ haemolytic toxicity vis$\grave{a}$-vis their preferential generation from the individual primaquine enantiomers are yet to be determined. Preferential formation of the orthoquinone marker of the $5-\mathrm{OH}-\mathrm{PQ}$, reported to be the most reactive primaquine metabolite, may explain the greater haemolytic effect of (+)-primaquine in rodent models [33-36]. However, it has been demonstrated in primates that the two enantiomers share identical radical curative potencies, though the toxicities are qualitatively different [22,24]. How the two enantiomers compare in efficacy and toxicity in humans is still a matter for further study. It was recently reported that when racemic primaquine is administered in a single dose to human volunteers, the carboxyprimaquine metabolite (quantitatively a major circulating metabolite) is virtually all derived from the (-)-primaquine [Walker et al. personal communication].

Understanding the pathways for metabolism of primaquine has been a daunting challenge. Two distinct pathways, the more prominent mono-amine oxidase-catalyzed generation of carboxyprimaquine, the major circulating metabolite, and another mediated through CYP are involved in the 
metabolism of primaquine. Clear evidence was obtained that specific CYP isoforms could accomplish the conversion of primaquine to methaemoglobin-generating metabolites $[8,35]$. Multiple hydroxylated metabolites were formed on in vitro incubation of primaquine with human hepatocytes and also CYP2D6 [16,18]. Hydroxylated metabolites of primaquine are known to be reactive in nature and have been shown to produce haemolytic toxicity $[8,29,33]$. Understanding the precise nature of the hydroxylated metabolites and the determination of their biological efficacy in vitro and in vivo has been challenging, primarily due to the reactive nature of these metabolites. This challenge has been addressed by the application of sensitive LC-MS/MS analytic methods, stable ${ }^{13} \mathrm{C}$-isotope labelled primaquine and highly reliable metabolite prediction software. Additionally, chemical synthesis of analytical and fully characterized hydroxylated analogs of primaquine, described herein, has provided further confirmation on the nature of several of the hydroxylated metabolites and their precise quantitative analysis.

Recently, CYP2D metabolism was demonstrated to be essential for the causal prophylactic efficacy of primaquine and several other 8-aminoquinoline compounds in mice $[19,20]$. The recent report by Bennett et al. also demonstrated the requirement for CYP2D6 metabolism for primaquine efficacy in humans [21]. These findings strongly suggest that a CYP2D6-generated metabolite(s) is responsible for the liver stage efficacy of primaquine. Although 5-OH-PQ has previously been suggested as the active metabolite [35-37], it has been difficult to generate conclusive evidence due to the highly reactive nature of this metabolite. The current studies, with chromatographic and mass spectrometric evidence identifying these primary hydroxylated species, afford new tools for probing these questions in vivo, and these studies are currently underway.

Differential pharmacological, toxicological, metabolism, and pharmacokinetic profiles of enantiomers of primaquine were recognized many years ago [22], but the findings in several laboratories suggest that these are highly species-dependent $[23,24]$. So far it has been difficult to substantially dissociate the efficacy and haemolytic effects of the enantiomers, although some suggestive evidence has been reported [22-24]. The current effort aimed to assess whether the enantiomers differ substantially in their ability to serve as substrates for recombinant human CYP2D6. In contrast to the markedly more prominent metabolism of (-)-primaquine, as compared to (+)-primaquine to carboxyprimaquine [25] (believed to proceed by sequential action of the amine oxidase and aldehyde dehydrogenase), (+)-primaquine was observed to be the preferred substrate for CYP2D6. The overall rate of metabolism of $(+)$-primaquine by human CYP2D6 was about 1.5 -fold faster compared to (-)-primaquine. Taken together, these findings suggest that (-)-primaquine should be less efficacious in humans, by virtue of the lower conversion to 5-OH-PQ marker (5,6 orthoquinone), and greater conversion to the carboxy metabolite, believed to be inactive. However, the ultimate fate of the carboxyprimaquine metabolite, which accumulates to high levels in plasma [14], is not clear. Recent findings suggest that carboxyprimaquine can be further metabolized in human hepatocytes to ring-hydroxylated metabolites [Walker et al. personal communication]. The pharmacological or toxicological significance of these, if any, remain to be elucidated. But given the basic structural requirements for CYP2D6 substrates, these are not likely generated via this pathway.

This study presents new evidence regarding different rates and metabolic profiles for the CYP2D6-mediated hydroxylation of primaquine and its enantiomers. It will be important to confirm the biological activities and metabolic profiles of the primaquine enantiomers in humans.

\section{Conclusion}

The metabolism of primaquine by human CYP2D6 and the generation of its metabolites display enantio-selectivity in the hydroxylated product profiles. (+)-Primaquine preferentially generated 2- and 5-OH-PQ while 3- and 4-OHPQ were predominantly generated from (-)-primaquine. This may partly explain differential pharmacologic and toxicologic properties of primaquine enantiomers. Genetic polymorphism of CYP2D6 in humans leading to the classification of slow and fast metabolizers may be an important consideration in primaquine therapy. The susceptibility of (+)-primaquine to CYP2D6 activity more than (-)-primaquine makes this important if individual enantiomers are administered.

\section{Abbreviations \\ G6PD: Glucose-6-phosphate dehydrogenase; NADPH: Nicotinamide adeninine dinucleotide phosphate; 2-OH-PQ: 2-hydroxyprimaquine; 3-OH-PQ: 3-hydroxyprimaquine; 4-OH-PQ: 4-hydroxyprimaquine; 5-OH-PQ: 5-hydroxyprimaquine; $\mathrm{OH}-\mathrm{PQ}$ : Hydroxyprimaquine; MAO: Monoamine oxidase; $\mathrm{MgCl}_{2}$ : Magnesium chloride; LC-MS/MS: Liquid chromatography-tandem mass spectrometry; UHPLC: Ultra-high performance liquid chromatography; IR: Infra-red; NMR: Nuclear magnetic resonance; TLC: Thin layer chromatography; $\mathrm{KOH}$ : Potassium hydroxide; $\mathrm{HCl}$ : Hydrochloric acid; RT: Retention time.}

\section{Competing interests}

The authors declare that they have no competing interests.

\section{Authors' contributions}

LAW, BLT, GAR, NPDN, and JDM developed the overall project direction; LAW, BSP, SRM, PSF, and BLT conceptualized the study; PSF, LAW and BLT designed the experiments; NPDN, HMTBH and VRA synthesized primaquine and its metabolites, PSF and SIK guided and performed the enzyme incubation studies and the sample preparation; PSF, BA, IAK, and YW performed the LC-MS analysis; PSF, LAW, JDM, MAE, and BLT performed data analysis and result interpretation. All authors contributed to the writing and the approval of the final version of the manuscript. All authors read and approved the final manuscript. 


\section{Acknowledgements}

This study is supported by Bill and Melinda Gates Foundation Phase I GCE Award OPP53288 and the US Army Medical Research and Materiel Command Awards Nos. W81XWH-07-2-0095 and W81XWH-10-2-0059 to the University of Mississippi. The authors thank Dr David Jollow, Medical University of South Carolina, retired, for his continuing input and advice and help in interpretation.

The views, opinions and/or findings contained in this presentation are those of the author(s) and do not necessarily reflect the views of the US Department of Defense and should not be construed as an official DoD/Army position, policy or decision unless so designated by other documentation. No official endorsement should be made.

\section{Author details}

${ }^{1}$ The National Center for Natural Products Research, University of Mississippi, University, MS 38677, USA. BioMolecular Sciences, University of Mississippi, University, MS 38677, USA. ${ }^{3}$ Pharmaceutical Sciences and Drug Delivery, School of Pharmacy, University of Mississippi, University, MS 38677, USA. ${ }^{4}$ EISohly Laboratories, Inc, 5 Industrial Park Dr, Oxford, MS38655USA. ${ }^{5}$ Military Malaria Research Program, Division of Experimental Therapeutics, Walter Reed Army Institute of Research, Silver Spring, MD 20910, USA. ${ }^{6}$ Ironstone Separations, Inc, Etta, MS 38627, USA.

Received: 15 October 2014 Accepted: 11 December 2014 Published: 17 December 2014

\section{References}

1. Tekwani BL, Walker LA: 8-aminoquinolines: future role as antiprotozoal drugs. Curr Opin Infect Dis 2006, 19:623-631.

2. Hill DR, Baird JK, Parise ME, Lewis LS, Ryan ET, Magill AJ: Primaquine: report from CDC expert meeting on malaria chemoprophylaxis. Am J Trop Med Hyg 2006, 75:402-415.

3. Vale N, Moreira R, Gomes P: Primaquine revisited six decades after its discovery. Eur J Med Chem 2009, 44:937-953.

4. Fernando D, Rodrigo C, Rajapakse S: Primaquine in vivax malaria: an update and review on management issues. Malar J 2011, 10:351.

5. WHO: Global Plan for Artemisinin Resistance Containment (GPARC). [http://www.wpro.who.int/mvp/artemisinin_resistance_containment_2011.pdf].

6. Beutler E, Duparc S: Glucose-6-phosphate dehydrogenase deficiency and antimalarial drug development. Am J Trop Med Hyg 2007, 77:779-789.

7. Uthman OA, Saunders R, Sinclair D, Graves P, Gelband H, Clarke A, Garner P: Safety of 8-aminoquinolines given to people with G6PD deficiency: protocol for systematic review of prospective studies. BMJ Open 2014, 4:e004664.

8. Tarlov AR, Brewer GL, Carson PE, Alving AS: Primaquine sensitivity. Arch Intern Med 1962, 109:209-234.

9. Avula B, Tekwani BL, Chaurasiya ND, Nanayakkara NP, Wang YH, Khan SI, Adelli VR, Sahu R, Elsohly MA, McChesney JD, Khan IA, Walker LA Profiling primaquine metabolites in primary human hepatocytes using UHPLC-QTOF-MS with 13C stable isotope labeling. J Mass Spectrom 2013, 48:276-285.

10. Allahyari R, Strother A, Fraser IM, Verbiscar AJ: Synthesis of certain hydroxy analogs of the antimalarial drug primaquine and their in vitro methemoglobin-producing and glutathione-depleting activity in human erythrocytes. J Med Chem 1984, 27:407-410.

11. Link CM, Theoharides AD, Anders JC, Chung H, Canfield CJ: Structure-activity relationships of putative primaquine metabolites causing methemoglobin formation in canine hemolysates. Toxicol Appl Pharmacol 1985, 81:192-202.

12. Fletcher KA, Barton PF, Kelly JA: Studies on the mechanisms of oxidation in the erythrocyte by metabolites of primaquine. Biochem Pharmacol $1988,37: 2683-2690$

13. Vasquez-Vivar J, Augusto O: Hydroxylated metabolites of the antimalarial drug primaquine: oxidation and redox cycling. J Bio Chem 1992, 267:6848-6854.

14. Mihaly GW, Ward SA, Edwards G, Orme ML, Breckenridge AM: Pharmacokinetics of primaquine in man: identification of the carboxylic acid derivative as a major plasma metabolite. Br J Clin Pharmacol 1984 17:441-446.

15. Peters $W$, Robinson $B L$ : The activity of primaquine and its possible metabolites against rodent malaria. In Primaquine: Pharmacokinetics,
Metabolism, Toxicity and Activity. Edited by Wernsdorfer WH, Trigg PI Chichester: John Wiley; 1984:93-101.

16. Ganesan S, Tekwani BL, Sahu R, Tripathi LM, Walker LA: Cytochrome P450dependent toxic effects of primaquine on human erythrocytes. Toxicol Appl Pharmacol 2009, 241:14-22.

17. Jin X, Pybus BS, Marcsisin SR, Logan T, Luong TL, Sousa J, Matlock N, Collazo V, Asher C, Carroll D, Olmeda R, Walker LA, Kozar MP, Melendez V: An LC-MS based study of the metabolic profile of primaquine, an 8-aminoquinoline antiparasitic drug, with an in vitro primary human hepatocyte culture model. Eur J Drug Metab Pharmacokinet 2014, 39:139-146.

18. Pybus BS, Sousa JC, Jin X, Ferguson JA, Christian RE, Barnhart R, Vuong C, Sciotti RJ, Reichard GA, Kozar MP, Walker LA, Ohrt C, Melendez V: CYP450 phenotyping and accurate mass identification of metabolites of the 8-aminoquinoline, anti-malarial drug primaquine. Malar J 2012, 11:259.

19. Bennett JW, Pybus BS, Yadava A, Tosh D, Sousa JC, McCarthy WF, Deye G, Melendez V, Ockenhouse CF: Primaquine failure and cytochrome P-450 2D6 in Plasmodium vivax malaria. N Engl J Med 2013, 369:1381-1382.

20. Marcsisin SR, Sousa JC, Reichard GA, Caridha D, Zeng Q, Roncal N, McNulty R, Careagabarja J, Sciotti RJ, Bennett JW, Zottig VE, Deye G, Li Q, Read L, Hickman M, Dhammika Nanayakkara NP, Walker LA, Smith B, Melendez V, Pybus BS: Tafenoquine and NPC-1161B require CYP 2D metabolism for anti-malarial activity: implications for the 8-aminoquinoline class of anti-malarial compounds. Malar J 2014, 13:2.

21. Pybus BS, Marcsisin SR, Jin X, Deye G, Sousa JC, Li Q, Caridha D, Zeng Q, Reichard GA, Ockenhouse C, Bennett J, Walker LA, Ohrt C, Melendez V: The metabolism of primaquine to its active metabolite is dependent on CYP 2D6. Malar J 2013, 12:212.

22. Schmidt LH, Alexander $S$, Allen L, Rasco J: Comparison of the curative antimalarial activities and toxicities of primaquine and its $\mathrm{d}$ and I isomers. Antimicrob Agents Chemother 1977, 12:51-60.

23. Nanayakkara ND, Tekwani BL, Herath HB, Sahu R, Gettayacamin M, Tungtaeng A, van Gessel Y, Baresel P, Wickham KS, Bartlett MS, Fronczek FR, Melendez V, Ohrt C, Reichard GA, McChesney JD, Rochford R, Walker LA: Scalable preparation and differential pharmacologic and toxicologic profiles of primaquine enantiomers. Antimicrob Agents Chemother 2014, 58:4737-4744

24. Brocks DR, Mehvar R: Stereoselectivity in the pharmacodynamics and pharmacokinetics of the chiral antimalarial drugs. Clin Pharmacokinet 2003, 42:1359-1382

25. Avula B, Khan SI, Tekwani BL, Nanayakkara NPD, McChesney JD, Walker LA, Khan IA: Analysis of primaquine and its metabolite carboxyprimaquine in biological samples: enantiomeric separation, method validation and quantification. Biomed Chromatogr 2011, 25:1010-1017.

26. Bortocan R, Bonato PS: Enantioselective analysis of primaquine and its metabolite carboxyprimaquine by capillary electrophoresis. Electrophoresis 2004, 25:2848-2853.

27. Herath HMT, McChesney JD, Walker LA, Nanayakkara NP: Synthesis of [13C6] primaquine. J Labelled Comp Radiopharm 2013, 56:341-343.

28. McChesney JD, Sarangan S: Synthesis of site specifically deuterated primaquines. II. $\mathrm{N}$-alkyl deuterated primaquines. J Label Compd Radiopharm 1984, 21:293-298.

29. LaMontagne MP, Markovac A, Menke JR: Antimalarials. 10. Synthesis of 4-substituted primaquine analogs as candidate antimalarials. J Med Chem 1977, 20:1122-1127.

30. Bowman ZS, Morrow JD, Jollow DJ, McMillan DC: Primaquine-induced hemolytic anemia: role of membrane lipid peroxidation and cytoskeletal protein alterations in the hemotoxicity of 5-hydroxyprimaquine. J Pharmacol Exp Ther 2005, 314:838-845.

31. Wetter WP, Blanton CD: 8-( $\omega$-Aminoalkylamino)quinolines as potential prophylactic antimalarials. J Med Chem 1974, 17:620-624.

32. Baker RH, Tinsley SW, Butler D, Riegel B: 3-Haloquinolines with substituents in the 6- and 8-positions. J Am Chem Soc 1950, 72:393-395.

33. Ganesan S, Chaurasiya ND, Sahu R, Walker LA, Tekwani BL: Understanding the mechanisms for metabolism-linked hemolytic toxicity of primaquine against glucose 6-phosphate dehydrogenase deficient human erythrocytes: evaluation of eryptotic pathway. Toxicology 2012, 294:54-60.

34. Bolchoz $\sqcup$, Budinsky RA, McMillan DC, Jollow DJ: Primaquine-induced hemolytic anemia: formation and hemotoxicity of the arylhydroxylamine metabolite 6-methoxy-8-hydroxylaminoquinoline. J Pharmacol Exp Ther 2001, 297:509-515. 
35. Liu H, Tekwani BL, Nanayakkara ND, Walker LA, Doerksen RJ: Methemoglobin generation by 8-aminoquinolines: effect of substitution at 5-position of primaquine. Chem Res Toxicol 2013, 26:1801-1809.

36. Bowman ZS, Oatis JE, Whelan JL, Jollow DJ, McMillan DC: Primaquine-induced hemolytic anemia: susceptibility of normal versus glutathione-depleted rat erythrocytes to 5-hydroxyprimaquine. J Pharmacol Exp Ther 2004, 309:79-85.

37. Bowman ZS, Jollow DJ, McMillan DC: Primaquine-induced hemolytic anemia: role of splenic macrophages in the fate of 5-hydroxyprimaquine-treated rat erythrocytes. J Pharmacol Exp Ther 2005, 315:980-986.

doi:10.1186/1475-2875-13-507

Cite this article as: Fasinu et al:: Enantioselective metabolism of

primaquine by human CYP2D6. Malaria Journal 2014 13:507.

\section{Submit your next manuscript to BioMed Central and take full advantage of:}

- Convenient online submission

- Thorough peer review

- No space constraints or color figure charges

- Immediate publication on acceptance

- Inclusion in PubMed, CAS, Scopus and Google Scholar

- Research which is freely available for redistribution 